\title{
Fatores associados à qualidade de vida de brasileiros e de diabéticos: evidências de um inquérito de base populacional
}

\author{
Factors associated with the quality of life of Brazilians and diabetic \\ patients: evidence from a population-based survey
}

Ranailla Lima Bandeira dos Santos ${ }^{1}$

Monica Rodrigues Campos ${ }^{1}$

Luisa Sório Flor ${ }^{1}$

${ }^{1}$ Escola Nacional de Saúde Pública Sérgio Arouca, Fiocruz. R. Leopoldo Bulhões 1480,

Manguinhos. 21041-210

Rio de Janeiro RJ Brasil.

ranaillalima@gmail.com

\begin{abstract}
This study sought to evaluate the associations between health conditions and sociodemographic characteristics in relation to quality of life (QoL) in the Brazilian population; as well as to assess the main factors associated with higher chances of a better QoL among diabetics. This is a study with data from a population-based survey conducted in Brazil in 2008. A total of 12,423 Brazilians aged $\geq 20$ years were analyzed, of which 935 were diabetic. QoL outcomes were measured using the summary components of the SF-36 instrument. QoL scores were classified as "above" and "below average" to obtain binary outcomes both for the general population and diabetics. Logistic regressions were performed to obtain prevalence ratios adjusted to the odds of above average QoL by adjusting for sociodemographic and health variables. Results indicate that female diabetics aged $\geq 65$ years old, with a sedentary lifestyle, belonging to $D / E$ social class and with more morbidities had greater chances of a worse physical and mental QoL. Regarding the general population, in addition to these factors, not having a partner and being illiterate were also responsible for worse QoL. Sedentary lifestyle and $D / E$ social class had greater influence for worse QoL among diabetics when compared to the general population.
\end{abstract}

Key words Diabetes mellitus, Quality of life, Sedentary lifestyle
Resumo Buscou-se avaliar as associações entre condições de saúde e características sociodemográficas em relação à qualidade de vida $(\mathrm{QV})$ na população brasileira; bem como estimar os principais fatores associados às chances de uma melhor QV entre diabéticos. Trata-se de um estudo com dados obtidos do inquérito de base populacional realizado no Brasil, em 2008. Foram analisados 12.423 brasileiros, com idade $\geq 20$ anos, cujos 935 eram diabéticos. Os desfechos de QV foram mensurados por meio dos componentes sumários do instrumento SF-36. Tanto para a população geral quanto para diabéticos, a $Q V$ teve seus escores agrupados em "acima" e "abaixo da média" para configurar desfechos binários. Foram realizadas regressões logísticas para obtenção das razões de prevalências ajustadas às chances de $Q V$ acima da média, controlando por variáveis sociodemográficas e de saúde. Resultados apontaram que as mulheres diabéticas com $\geq 65$ anos, sedentárias, pertencentes à classe D/E e com mais morbidades apresentam maiores chances de uma pior $Q V$ fisica e mental. Para a população geral além desses fatores, não ter um companheiro e ser analfabeto proporcionou uma pior QV. O sedentarismo e a classe D/E apresentaram maior influência para pior QV entre diabéticos quando comparado à população total.

Palavras-chave Diabetes mellitus, Qualidade de vida, Estilo de vida sedentário 


\section{Introdução}

O Diabetes mellitus (DM) é definido como uma síndrome de etiologia múltipla e pode ser ocasionado pela falta de insulina e/ou incapacidade de a insulina exercer adequadamente suas funções. Quando não controlado, o DM pode levar ao desenvolvimento de complicações microvasculares, como retinopatia, nefropatia e neuropatia, e macrovasculares, como infarto agudo do miocárdio ${ }^{1}$.

O DM é uma doença onerosa, de ocorrência frequente e incidência crescente em todo o mundo e de acordo com cálculos estimados para 2015 pela Internacional Diabetes Federation (IDF), a prevalência de DM foi 415 milhões, representando $8,8 \%$ da população mundial. As projeções para o ano de 2040 apontam que 642 milhões de pessoas $(10,4 \%)$ terão $\mathrm{DM}$, constituindo assim um problema de saúde pública em potencial ${ }^{2}$.

No Brasil, dados recentes da Pesquisa Nacional em Saúde (PNS) de 2013 estimou a prevalência de DM em 6,2\% dos participantes com 18 anos ou mais ${ }^{3}$. De acordo com a IDF, o Brasil ocupa a quarta posição entre os países com o maior número de diabéticos, cerca de $14,3 \mathrm{mi}-$ lhões em $2015^{2}$.

O envelhecimento populacional, a crescente urbanização, o sedentarismo, as dietas hipercalóricas e a obesidade são os grandes fatores de risco responsáveis pelo aumento da prevalência de diabetes mellitus, configurando-o, como uma epidemia mundial ${ }^{4}$. Ao todo, 5 milhões de mortes por ano no mundo são determinadas por essa doença e suas complicações, representando 9\% do total de mortes ${ }^{2}$.

Diversos fatores podem influenciar a qualidade de vida (QV) dos diabéticos. Entre esses, a idade, o sexo, a obesidade, a presença de complicações e o esquema terapêutico antidiabético ${ }^{5}$. Além disso, quanto mais graves forem as complicações de um paciente com DM, pior tende a ser sua $\mathrm{QV}^{6}$.

Por esse motivo, torna-se fundamental a avaliação da QV das pessoas com diabetes mellitus, sendo necessária a identificação de fatores que interferem na QV, para que possam ser instituídas estratégias de cuidado específicas e efetivas na minimização ou prevenção do seu comprometimento ${ }^{7}$.

Em decorrência da elevada incidência e prevalência do diabetes mellitus e suas complicações crônicas, além de serem limitados os estudos de base populacional no Brasil que propõem investigar a prevalência de DM e medir simultanea- mente $\mathrm{QV}$, os resultados desse trabalho merecem destaque pois, além de originarem-se de um estudo de base populacional, possuem representatividade nacional. Devido a abrangência da pesquisa, foi possível lidar com um número expressivo de casos de DM e uma ampla gama de variáveis pôde ser associada à ocorrência desse agravo.

O presente trabalho tem como objetivo estimar a magnitude e direção das associações existentes entre condições de saúde, incluindo o DM e características sociodemográficas em relação aos escores de QV física e mental na população brasileira; bem como, estimar os principais fatores associados às chances de uma melhor $\mathrm{QV}$ física e mental, em específico para os indivíduos com diagnóstico de diabetes mellitus.

\section{Metodologia}

\section{Estudo fonte}

Trata-se de um estudo analítico transversal pautado nos dados provenientes do survey nacional, denominado "Pesquisa Dimensões Sociais das Desigualdades" (PDSD), conduzido em 2008. O projeto, coordenado pelo Instituto Universitário de Pesquisas do Rio de Janeiro (IUPERJ), buscou entrevistar chefes de famílias e cônjuges, ambos com idade superior a 20 anos, a fim de produzir informações sobre as diversas dimensões das desigualdades sociais e compreender o mecanismo de produção e reprodução da desigualdade ao longo do ciclo de vida. Para isso, investigou temas relevantes, tais como, condições de vida, educação, ocupação e saúde ${ }^{8}$. A PDSD foi submetida ao Comitê de Ética em Pesquisa da Escola Nacional de Saúde Pública Sergio Arouca e sua realização foi considerada adequada à população humana. O banco de dados encontrase com acesso livre e irrestrito e disponível para download ${ }^{9}$.

\section{Amostra}

A população estudada foi determinada por meio de amostragem aleatória estratificada em múltiplos estágios, o que permite inferências acuradas dos parâmetros populacionais. A amostragem segue um procedimento probabilístico em três etapas: na primeira foram selecionados os municípios, na segunda os setores censitários dentro dos municípios, e na terceira os domicílios dentro dos setores. A amostra foi constituída por 1.374 setores censitários (unidades primárias 
amostrais) e 8.048 domicílios particulares permanentes (unidades secundárias amostrais) em setores comuns ou não especiais, inclusive favelas em áreas urbanas e rurais de todas as regiões do Brasil. Cerca de 20\% dos domicílios primários foram substituídos por outros, principalmente dada à recusa ou impossibilidade de um dos cônjuges em receber o entrevistador. Ao final, 12.423 participantes adultos de ambos os sexos foram entrevistados ${ }^{8}$.

\section{Instrumento - The Short Form Health Survey (SF-36)}

A QV foi mensurada por meio do questionário SF-36, v.2, tipo standard, onde as perguntas foram relativas às últimas quatro semanas prévias à entrevista. O SF-36 foi desenvolvido no final dos anos 1980 nos Estados Unidos e foi traduzido e validado no Brasil ${ }^{10}$ e mostrou-se adequado às condições socioeconômicas e culturais da população brasileira. Além disso, o fato de ser um instrumento versátil e que permite comparação internacional de resultados torna o SF-36 uma atraente ferramenta para uso nos inquéritos populacionais ${ }^{11}$. A PDSD usou este instrumento, pois ele demonstra boa consistência interna, validade de construto e confiabilidade ${ }^{8}$.

O SF-36 é classificado como instrumento genérico de medida de qualidade de vida, ou seja, avalia o estado de saúde e é utilizado para avaliar diversos domínios aplicáveis aos diferentes estados de saúde de distintos tipos de população ou doenças. É multidimensional, possui ampla aplicabilidade e permite comparar a QV de indivíduos sadios com a de indivíduos doentes ou de portadores da mesma doença vivendo em diferentes contextos sociais e culturais. Porém, não é sensível na detecção de aspectos particulares e específicos da QV decorrentes de uma determinada patologia ${ }^{12}$.

O SF-36 é composto por 36 itens ou questões que avaliam oito domínios de saúde: Capacidade funcional (desempenho das atividades diárias, como capacidade de cuidar de si, vestir-se, tomar banho e subir escadas); Aspectos físicos (impacto da saúde física no desempenho das atividades diárias e ou profissionais); Dor corporal (nível de dor e o impacto no desempenho das atividades diárias e ou profissionais); Estado geral de saúde (percepção subjetiva do estado geral de saúde); Vitalidade (percepção subjetiva do estado de saúde); Aspectos sociais (reflexo da condição de saúde física nas atividades sociais); Aspectos emocionais (reflexo das condições emocionais no desempenho das atividades diárias e ou profissionais); Saúde mental (escala de humor e bem-estar) e uma questão comparativa sobre a percepção atual da saúde e há um ano ${ }^{13}$.

Para o estudo, foram consideradas as medidas sumárias: Componente físico - PCS (phisical component summary) e Componente mental - MCS (mental componente summary). Essas dimensões, bem como os oito domínios do SF-36, são pontuadas em uma escala de 0 a 100, sendo 100 o melhor estado de saúde possível. Esses escores foram, entretanto, transformados em escores normalizados em relação à população americana, com média de 50 e desvio padrão de 10 . Dessa forma, toda vez que a pontuação de uma escala encontra-se menor que 50, o estado de saúde está abaixo da média ${ }^{13}$.

\section{Variáveis de estudo}

Para a análise da amostra total do estudo ( $\mathrm{n}$ = 12.423; Tabelas 1, 2 e 3), foram adotadas como variáveis dependentes os escores de QV: PCS acima da média (PCS > 50) e MCS acima da média (MCS > 50). E, para a análise da população de diabéticos ( $\mathrm{n}=935$; Tabelas 4 e 5$)$ as variáveis dependentes foram PCS acima da média (PCS $>$ 42) e MCS acima da média (MCS > 47).

No que tange às variáveis independentes, abordaram-se variáveis sociodemográficas; comportamentais e de condições de saúde. No contexto sociodemográfico foram incluídas as variáveis sexo (masculino ou feminino); idade, em anos completos, categorizada por faixa etária (20-39; 40-64; $\geq 65$ anos) e raça (pardo/preto ou branco). O nível de escolaridade foi classificado em cinco categorias segundo anos completos na escolaridade formal (analfabeto; 1-4 anos; 5-8 anos; 9-11 anos; 12 anos ou mais). A variável estado civil foi classificada em duas categorias: casado/união e outros. Por fim, a classe econômica foi definida segundo o Critério de Classificação Econômica do Brasil $2008^{14}$ e agregada para fins de análise em três categorias (A/B; C; D/E).

Quanto às variáveis comportamentais e de condições de saúde, os indivíduos foram classificados segundo a presença de pelo menos uma morbidade crônica exceto Diabetes mellitus (sim ou não) e a presença/ausência de Diabetes mellitus, ambos por meio de diagnóstico prévio autorreferido. Por fim, o sedentarismo - no presente ou no passado - foi expresso em duas categorias ( $\operatorname{sim}$ ou não), onde "Sedentário = sim", significa: indivíduo "fica sentado a maior parte do dia" ou "não anda muito durante o dia"15. 
Apesar da análise de Diabetes mellitus incorporar portadores do DM tipo 1 e DM tipo 2, sabe-se, pela literatura, que o DM tipo 2 chega a corresponder por mais de $90 \%$ de todos os casos de DM, tornando-se este o foco principal desse trabalho².

\section{Análise dos dados}

$\mathrm{Na}$ análise dos dados da amostra total $(\mathrm{n}=$ 12.423), foram utilizadas estatísticas descritivas para os componentes de QV física (PCS) e mental (MCS) segundo características sociodemográficas, condições de saúde e comportamentais dos brasileiros (variáveis independentes), utilizando-se em todas as etapas da análise os pesos amostrais correspondentes ao desenho da amostra estratificada. Os resultados foram expostos em tabela com medidas de frequência absoluta e relativa para as variáveis categóricas. Para as medidas contínuas sumárias de QV do SF-36 (escores de PCS e MCS), segundo as variáveis independentes citadas acima, foram apresentadas ainda as médias e desvios padrão, bem como realizados os testes de diferença de médias, Teste $t$ ou ANOVA, conforme apropriado, ao nível de significância de 5\%.

Para verificar a associação entre as variáveis de exposição (independentes: sociodemográficas, comportamentais e de saúde) e os desfechos de QV física e mental acima da média, tanto para a amostra total $(\mathrm{n}=12.423)$, quanto apenas entre os entrevistados que se auto referiram diagnóstico clínico prévio de DM ( $\mathrm{n}=935)$, utilizou-se o teste Chi-quadrado de Pearson $\left({ }^{2}\right)$, sendo consideradas significativas as variáveis que apresentaram valor $\mathrm{p}<0,05$, sendo apresentadas as prevalências dos desfechos (e respectiva significância estatística - p-valor) em cada categoria de exposição analisada.

Em etapa subsequente, utilizou-se o modelo de regressão logística multivariada, cuja escolha buscou adequar a necessidade de controle de múltiplas variáveis de confusão e o uso, como variável resposta, de um evento binário. O método de seleção de variáveis utilizado foi o stepwise backward. Foram incluídas no modelo as variáveis que apresentaram significância estatística na análise bivariada ( $p$-valor $<5 \%$ ) e, após as interações, foram eliminadas do modelo final multivariado as variáveis com $p>0,05$. Determinou-se, por fim, as razões de chances (OR) ajustadas - exponencial dos coeficientes de regressão logística - e seus intervalos de confiança a 95\%, bem como as probabilidades preditas para o desfecho, segundo variáveis significativas no modelo final. A proporção de classificação correta entre os positivos e entre os negativos para o desfecho e a proporção de classificação total (overall), foram também determinadas.

Ratifica-se que para ambos os grupos investigados (população geral e diabéticos), foram apresentados, ainda, para efeito de comparação, os OR brutos, obtidos na análise bivariada dos desfechos e variáveis independentes calculados diretamente quando estas eram binárias e via regressão logística univariada, quando necessário - exposição com mais de duas categorias, por exemplo, classe econômica.

Os dados foram analisados por meio do software Statistical Package for the Social Sciences (SPSS) for Windows, versão $17.0^{16}$.

\section{Resultados}

$\mathrm{Na}$ amostra do estudo, 7.168 (57,7\%) dos respondentes são do sexo feminino, a média de idade é de 48,5 anos (DP $=16$ ), $48,7 \%$ se auto classificaram como brancos, $53 \%$ tinham cursado de um a oito anos de estudo, 71\% estavam casados ou viviam com companheiro (a), 46,6\% pertenciam a classe econômica " $C$ ", $29,8 \%$ foram classificados como sedentários, $63,3 \%$ relataram ter alguma morbidade crônica e 7,5\% têm DM (Tabela 1).

Em relação ao diabetes mellitus, percebe-se que o grupo acometido com a doença apresenta piores médias de escores de PCS $(42,03)$ e MCS $(47,13)$, enquanto os não acometidos apresentam 49,92 de PCS e 51,38 de MCS. O mesmo foi percebido para os sedentários, onde a QV é menor para quem é sedentário, com PCS médio de 45,73 e MCS médio de 48,62, enquanto que para os não sedentários eram 50,88 e 52,13 respectivamente (Tabela 1).

Ainda no que diz respeito à $\mathrm{QV}$, homens apresentaram melhor saúde física e mental que as mulheres. Com o avançar da idade percebese que PCS e MCS diminuem, sendo a QV física mais afetada. Porém, ao aplicar-se o Teste $t$ para a variável raça não verificou-se diferença de média estatisticamente significativa no tocante à $\mathrm{QV}$ ( $\mathrm{p}$ valor $>0,05)$. Os casados tiveram melhor pontuação para PCS e MCS. Percebe-se que a melhora na QV é diretamente proporcional ao aumento da escolaridade, sendo este padrão mais evidente na QV física. Indivíduos com 12 anos ou mais de estudo apresentaram escore médio do PCS de 
Tabela 1. Análise descritiva dos componentes de qualidade de vida física (PCS) e mental (MCS) segundo características sociodemográficas e condições de saúde. Brasil, 2008.

\begin{tabular}{|c|c|c|c|c|c|c|}
\hline \multirow{2}{*}{ Variáveis $\left.{ }^{*}\right)$} & \multirow{2}{*}{$\mathbf{N}$} & \multirow{2}{*}{$\%$} & \multicolumn{2}{|c|}{ PCS - Componente Físico } & \multicolumn{2}{|c|}{ MCS - Componente Mental } \\
\hline & & & Média & Desvio Padrão & Média & Desvio Padrão \\
\hline \multicolumn{7}{|l|}{ Sexo } \\
\hline Masculino & 5255 & 42,3 & 50,67 & 10,49 & 52,87 & 10,12 \\
\hline Feminino & 7168 & 57,7 & 48,34 & 11,07 & 49,73 & 11,68 \\
\hline \multicolumn{7}{|l|}{ Idade (anos) } \\
\hline 20-39 & 3973 & 32,0 & 54,34 & 7,86 & 52,05 & 9,96 \\
\hline $40-64$ & 6132 & 49,4 & 48,93 & 10,58 & 50,77 & 11,39 \\
\hline$\geq 65$ & 2318 & 18,7 & 41,77 & 11,53 & 50,13 & 12,31 \\
\hline \multicolumn{7}{|l|}{ Raça } \\
\hline Pardo/ Preto & 6190 & 51,3 & 49,26 & 10,88 & 51,01 & 11,04 \\
\hline Branco & 5868 & 48,7 & 49,40 & 10,90 & 51,17 & 11,18 \\
\hline \multicolumn{7}{|l|}{ Estado civil } \\
\hline Casado/ União & 8823 & 71,0 & 49,97 & 10,56 & 51,78 & 10,70 \\
\hline Outros & 3601 & 29,0 & 47,74 & 11,51 & 49,31 & 12,04 \\
\hline \multicolumn{7}{|l|}{ Anos de estudo } \\
\hline Analfabeto & 1904 & 16,5 & 43,21 & 12,13 & 48,06 & 12,81 \\
\hline $1-4$ & 3592 & 31,1 & 47,48 & 11,20 & 50,66 & 11,45 \\
\hline $5-8$ & 2529 & 21,9 & 50,87 & 10,12 & 51,24 & 11,06 \\
\hline $9-11$ & 2408 & 20,9 & 53,06 & 8,67 & 52,77 & 9,79 \\
\hline$\geq 12$ & 1109 & 9,6 & 53,04 & 8,43 & 53,10 & 9,65 \\
\hline \multicolumn{7}{|l|}{ Classe econômica } \\
\hline $\mathrm{A} / \mathrm{B}$ & 2390 & 19,2 & 52,41 & 8,64 & 53,35 & 9,25 \\
\hline $\mathrm{C}$ & 5788 & 46,6 & 49,73 & 10,63 & 51,17 & 11,13 \\
\hline $\mathrm{D} / \mathrm{E}$ & 4244 & 34,2 & 47,04 & 11,84 & 49,63 & 11,93 \\
\hline \multicolumn{7}{|l|}{ Sedentarismo (\#) } \\
\hline Não & 8641 & 70,2 & 50,88 & 9,70 & 52,13 & 10,47 \\
\hline Sim & 3655 & 29,8 & 45,73 & 12,54 & 48,62 & 12,26 \\
\hline \multicolumn{7}{|c|}{ Número de morbidades crônicas } \\
\hline 0 & 4554 & 36,7 & 54,93 & 7,27 & 54,02 & 8,53 \\
\hline 1 & 3035 & 24,4 & 50,33 & 9,85 & 52,09 & 10,10 \\
\hline 2 & 1996 & 16,1 & 46,38 & 10,90 & 50,36 & 11,77 \\
\hline$\geq 3$ & 2837 & 22,8 & 41,32 & 11,19 & 45,71 & 13,34 \\
\hline \multicolumn{7}{|l|}{ Diabetes mellitus } \\
\hline Não & 11489 & 92,5 & 49,92 & 10,64 & 51,38 & 10,98 \\
\hline Sim & 935 & 7,5 & 42,03 & 11,24 & 47,13 & 12,55 \\
\hline Total & 12423 & 100,0 & 49,32 & 10,89 & 51,06 & 11,16 \\
\hline
\end{tabular}

${ }^{*}$ ) Todas as variáveis investigadas apresentaram diferença de média estatisticamente significativa (p-valor $\left.<5 \%\right)$ tanto para o PCS quanto para o MCS, entre as categorias testadas via Teste tou ANOVA quando aplicável, exceto a variável raça. (\#) Sedentário: indivíduo "fica sentado a maior parte do dia" ou "não anda muito durante o dia". Nota: Com aplicação de pesos amostrais correspondentes ao desenho da amostra estratificada, sendo os "n" originais e os percentuais ponderados.

53,04 , enquanto para analfabetos esse valor foi de 43,21. E quanto pior o status de classe econômica menor é a QV (Tabela 1).

Ressalta-se o gradiente monotônico decrescente nos escore de QV, no PCS e no MCS, segundo as categorias de quantidade de morbidades crônicas autorreferidas (ausência; uma mor- bidade; duas; três ou mais - todos com p-valor $<5 \%$ ):54,93; 50,33; 46,38 e 41,32 para o PCS e 54,$02 ; 52,09 ; 50,36$ e 45,71 para o MCS, respectivamente (Tabela 1 ).

O efeito da variável sexo para a QV acima da média é mais acentuado entre os diabéticos (Tabela 4) do que para a população geral (Tabela 2). 
Tabela 2. Distribuição da amostra de adultos ( $\geq 20$ anos) ( $n=12.423)$, segundo variáveis sociodemográficas, comportamentais e de saúde, por situação de qualidade de vida física e mental acima da média $(>50)$. Brasil, 2008.

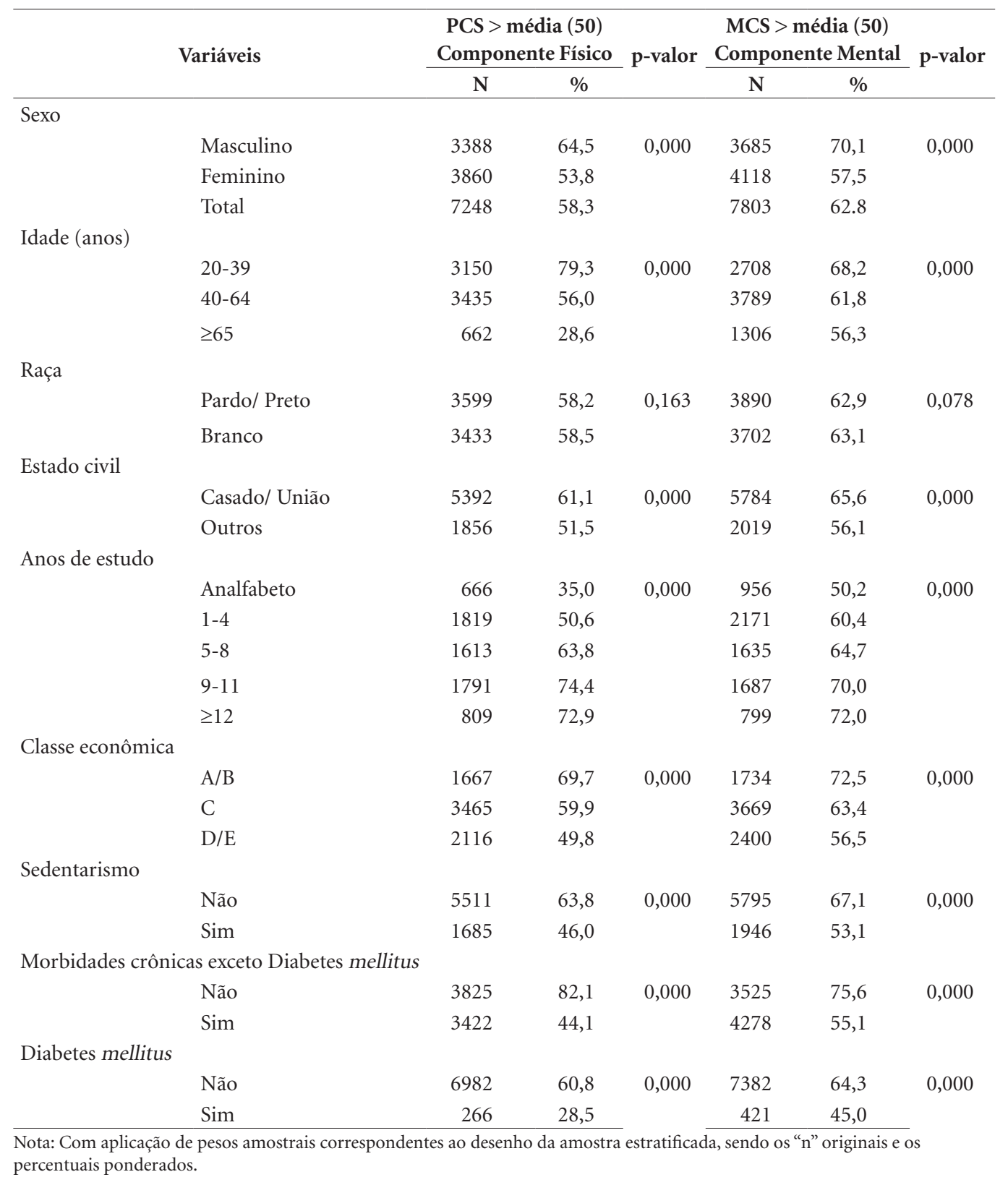

Observa-se que os homens têm uma potencialização para uma melhor QV, em ambos os estratos analisados.

Observou-se que quanto maior a idade menor a proporção de indivíduos com QV acima da média (Tabelas 2 e 4) em cada faixa etária. Sobre este aspecto, destaca-se que na população geral, a idade apresentou associação com o PCS e MCS acima da média, porém para o MCS os valores de OR são limítrofes e tem efeito inverso do PCS, ou seja, aqueles com "65 anos ou mais” possuem maiores chances de ter uma melhor QV mental quando comparados com quem tem "20 a 40 anos" (Tabela 3). Ao analisar as chances de obter uma boa QV, em relação aos grupos etários, verifica-se que a magnitude do OR bruto altera-se de 10 para 4, quando controlado pelas demais variáveis (Tabela 3). Esse cenário é igualmente verifi- 
Tabela 3. Odds ratio (OR) bruto e ajustado no modelo final de regressão logística multivariada (stepwise backward) na amostra de adultos (>20 anos) (n=12.423), com desfechos "ter qualidade de vida acima da média": domínio físico (PCS > 50) e domínio mental (MCS > 50). Brasil, 2008.

\begin{tabular}{|c|c|c|c|c|c|c|c|c|c|c|}
\hline \multirow{3}{*}{$\begin{array}{c}\text { Regressão } \\
\text { Logística Stepwise } \\
\text { Backward }\end{array}$} & \multicolumn{5}{|c|}{ PCS acima da média $(\mathrm{PCS}>50)$} & \multicolumn{5}{|c|}{ MCS acima da média $($ MCS $>50)$} \\
\hline & \multirow{2}{*}{$\begin{array}{l}\text { Odds } \\
\text { ratio } \\
\text { bruto }\end{array}$} & \multirow{2}{*}{$\begin{array}{c}\text { Odds } \\
\text { ratio } \\
\text { ajustado }\end{array}$} & \multicolumn{2}{|c|}{$\begin{array}{c}\text { IC - } 95 \% \\
\text { OR Ajustado }\end{array}$} & \multirow{2}{*}{ p-valor } & \multirow{2}{*}{$\begin{array}{c}\text { Odds } \\
\text { ratio } \\
\text { bruto }\end{array}$} & \multirow{2}{*}{$\begin{array}{c}\text { Odds } \\
\text { ratio } \\
\text { ajustado }\end{array}$} & \multicolumn{2}{|c|}{$\begin{array}{c}\text { IC - 95\% } \\
\text { OR Ajustado }\end{array}$} & \multirow{2}{*}{ p-valor } \\
\hline & & & $\begin{array}{l}\text { Limite } \\
\text { inferior }\end{array}$ & $\begin{array}{l}\text { Limite } \\
\text { superior }\end{array}$ & & & & $\begin{array}{l}\text { Limite } \\
\text { inferior }\end{array}$ & $\begin{array}{c}\text { Limite } \\
\text { superior }\end{array}$ & \\
\hline \multicolumn{11}{|l|}{ Sexo } \\
\hline Masculino & 1,56 & 1,44 & 1,31 & 1,58 & 0,000 & 1,74 & 1,51 & 1,38 & 1,64 & 0,000 \\
\hline Feminino $^{(\&)}$ & 1,00 & 1,00 & - & - & - & 1,00 & 1,00 & - & - & - \\
\hline \multicolumn{11}{|l|}{ Idade (anos) } \\
\hline $20-39^{(\&-P C S)}$ & 9,57 & 4,20 & 3,63 & 4,86 & 0,000 & 1,00 & 1,00 & - & - & - \\
\hline $40-64$ & 3,18 & 2,10 & 1,87 & 2,37 & 0,000 & 1,66 & 1,08 & 0,97 & 1,19 & 0,157 \\
\hline$\geq 65^{(\&-M C S)}$ & 1,00 & 1,00 & - & - & - & 1,25 & 1,30 & 1,14 & 1,49 & 0,000 \\
\hline \multicolumn{11}{|l|}{ Estado civil } \\
\hline Casado/ União & 1,48 & 1,11 & 1,01 & 1,23 & 0,041 & 1,50 & 1,21 & 1,10 & 1,33 & 0,000 \\
\hline Outros ${ }^{(\&)}$ & 1,00 & 1,00 & - & - & - & 1,00 & 1,00 & - & - & - \\
\hline \multicolumn{11}{|l|}{ Anos de estudo } \\
\hline Analfabeto $^{(\&)}$ & 1,00 & 1,00 & - & - & - & 1,00 & 1,00 & - & - & - \\
\hline $1-4$ & 1,90 & 1,56 & 1,36 & 1,78 & 0,000 & 1,51 & 1,40 & 1,24 & 1,58 & 0,000 \\
\hline $5-8$ & 3,27 & 1,82 & 1,57 & 2,12 & 0,000 & 1,81 & 1,50 & 1,30 & 1,73 & 0,000 \\
\hline $9-11$ & 5,39 & 2,50 & 2,11 & 2,96 & 0,000 & 2,31 & 1,69 & 1,44 & 1,97 & 0,000 \\
\hline$\geq 12$ & 5,00 & 2,82 & 2,27 & 3,51 & 0,000 & 2,55 & 1,90 & 1,55 & 2,32 & 0,000 \\
\hline \multicolumn{11}{|l|}{ Classe econômica } \\
\hline $\mathrm{A} / \mathrm{B}$ & 2,32 & 1,64 & 1,40 & 1,92 & 0,000 & 2,03 & 1,59 & 1,37 & 1,84 & 0,000 \\
\hline $\mathrm{C}$ & 1,50 & 1,21 & 1,09 & 1,34 & 0,000 & 1,33 & 1,16 & 1,05 & 1,28 & 0,003 \\
\hline $\mathrm{D} / \mathrm{E}^{(\&)}$ & 1,00 & 1,00 & - & - & - & 1,00 & 1,00 & - & - & - \\
\hline \multicolumn{11}{|l|}{ Sedentarismo } \\
\hline Não & 2,07 & 1,89 & 1,71 & 2,08 & 0,000 & 1,80 & 1,72 & 1,57 & 1,87 & 0,000 \\
\hline $\operatorname{Sim}^{(\&)}$ & 1,00 & 1,00 & - & - & - & 1,00 & 1,00 & - & - & - \\
\hline \multicolumn{11}{|c|}{$\begin{array}{l}\text { Morbidades crônicas } \\
\text { exceto Diabetes mellitus }\end{array}$} \\
\hline Não & 5,78 & 4,32 & 3,91 & 4,78 & 0,000 & 2,53 & 2,34 & 2,13 & 2,56 & 0,000 \\
\hline $\operatorname{Sim}^{(\&)}$ & 1,00 & 1,00 & - & - & - & 1,00 & 1,00 & - & - & - \\
\hline \multicolumn{11}{|l|}{ Diabetes mellitus } \\
\hline Não & 3,89 & 1,97 & 1,67 & 2,34 & 0,000 & 2,20 & 1,46 & 1,46 & 1,96 & 0,000 \\
\hline $\operatorname{Sim}^{(\&)}$ & 1,00 & 1,00 & - & - & - & 1,00 & 1,00 & - & - & - \\
\hline
\end{tabular}

${ }^{(\&)}$ Categoria de referência da razão de chance (OR). Nota: Com aplicação de pesos amostrais correspondentes ao desenho da amostra estratificada, sendo os " $n$ " originais e os percentuais ponderados.

cado entre os diabéticos, embora de forma mais atenuada (Tabela 5). Para os diabéticos a idade não se mostra associada ao MCS no modelo final (Tabela 5).

Na população geral, "ter um companheiro" potencializa a boa QV física e mental (Tabelas $2 \mathrm{e}$ 3), o que não foi encontrado entre os diabéticos (Tabela 4). Para estes, o estado civil só indicou melhora da QV mental na abordagem descritiva 
Tabela 4. Distribuição da amostra de diabéticos $(n=935)$, segundo variáveis sociodemográficas, comportamentais e de saúde em adultos ( $\geq 20$ anos) por situação de qualidade de vida física acima da média (PCS > 42) e mental (MCS > 47). Brasil, 2008.

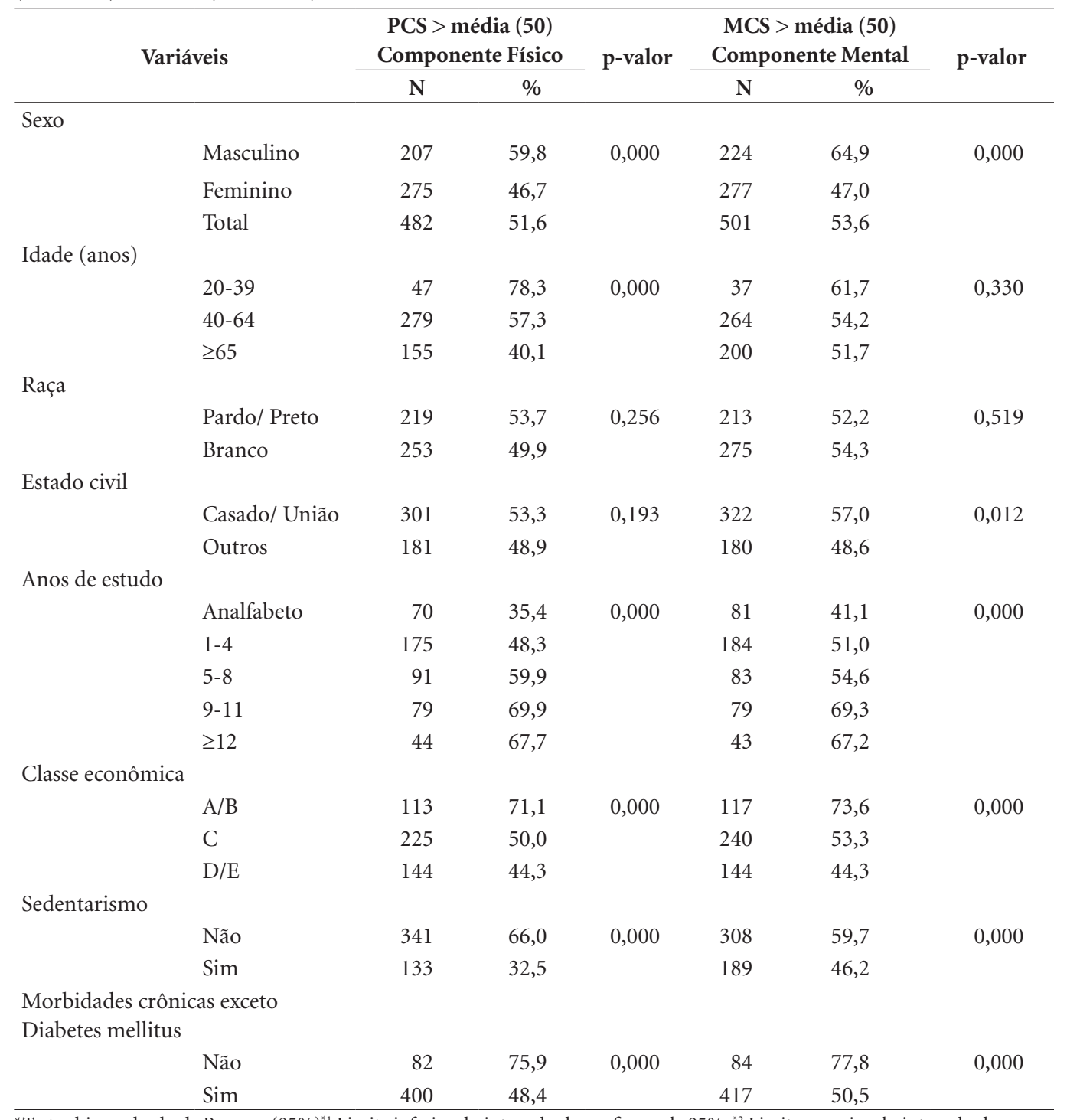

*Teste chi-quadrado de Pearson $(95 \%)^{* 1}$ Limite inferior do intervalo de confiança de $95 \%$; ${ }^{* 2}$ Limite superior do intervalo de confiança de 95\%. Nota: Com aplicação de pesos amostrais correspondentes ao desenho da amostra estratificada, sendo os " $n$ " originais e os percentuais ponderados.

gradiente observado se mantenha. Ou seja, quanto mais anos de estudo, maiores são as chances de obter-se QV acima da média (Tabela 3).

Destaca-se que a proporção de indivíduos com QV acima da média aumenta nas classes econômicas mais favorecidas, tanto na população geral (Tabela 2), quanto entre os diabéticos, sendo mais acentuado seu efeito para os portadores de DM, principalmente na QV mental (Tabela 4). $\mathrm{O}$ efeito da classe econômica na QV física e mental é cerca de duas vezes maior entre os diabéticos (OR ajustado para o PCS $=2,98$; OR ajustado para o MCS $=3,24)($ Tabela 5), quando comparado ao efeito na população geral (OR ajustado para o PCS $=1,64$; OR ajustado para o MCS = 1,59) (Tabela 3). Assim, a QV mental é mais afetada nos diabéticos pertencentes às classes $\mathrm{D} / \mathrm{E}$ (Tabela 5), que aqueles pertencentes à população geral.

Como apontado anteriormente para "classe econômica", o sedentarismo exerceu um forte impacto na piora da $\mathrm{QV}$, principalmente nos diabéticos. Porém, ressalta-se que a magnitude do efeito deste na piora da QV se dá de maneira mais 
Tabela 5. Odds ratio (OR) bruto e ajustado no modelo final de regressão logística multivariada (stepwise backward) na amostra de diabéticos ( $\mathrm{n}=935)$, com desfechos "ter qualidade de vida acima da média”: domínio físco (PCS > 42) e domínio mental (MCS > 47). Brasil, 2008.

\begin{tabular}{|c|c|c|c|c|c|c|c|c|c|c|}
\hline \multirow{3}{*}{$\begin{array}{c}\text { Regressão } \\
\text { Logística } \\
\text { Stepwise } \\
\text { Backward }\end{array}$} & \multicolumn{5}{|c|}{ PCS acima da média $(P C S>42)$} & \multicolumn{5}{|c|}{ MCS acima da média $($ MCS $>47)$} \\
\hline & \multirow{2}{*}{$\begin{array}{l}\text { Odds } \\
\text { ratio } \\
\text { bruto }\end{array}$} & \multirow{2}{*}{$\begin{array}{c}\text { Odds } \\
\text { ratio } \\
\text { ajustado }\end{array}$} & \multicolumn{2}{|c|}{$\begin{array}{c}\text { IC - } 95 \% \\
\text { OR Ajustado }\end{array}$} & \multirow{2}{*}{ p-valor } & \multirow{2}{*}{$\begin{array}{l}\text { Odds } \\
\text { ratio } \\
\text { bruto }\end{array}$} & \multirow{2}{*}{$\begin{array}{c}\text { Odds } \\
\text { ratio } \\
\text { ajustado }\end{array}$} & \multicolumn{2}{|c|}{$\begin{array}{c}\text { IC - } 95 \% \\
\text { OR Ajustado }\end{array}$} & \multirow{2}{*}{ p-valor } \\
\hline & & & $\begin{array}{l}\text { Limite } \\
\text { inferior }\end{array}$ & $\begin{array}{l}\text { Limite } \\
\text { superior }\end{array}$ & & & & $\begin{array}{l}\text { Limite } \\
\text { inferior }\end{array}$ & $\begin{array}{l}\text { Limite } \\
\text { superior }\end{array}$ & \\
\hline \multicolumn{11}{|l|}{ Sexo } \\
\hline Masculino & 1,70 & 1,76 & 1,28 & 2,43 & 0,000 & 2,08 & 1,87 & 1,39 & 2,53 & 0,000 \\
\hline Feminino $^{(\&)}$ & 1,00 & 1,00 & - & - & - & 1,00 & 1,00 & - & - & - \\
\hline \multicolumn{11}{|l|}{ Idade (anos) } \\
\hline 20-39 & 5,59 & 3,18 & 1,56 & 6,49 & 0,001 & 1,54 & - & - & - & - \\
\hline $40-64$ & 1,99 & 1,44 & 1,06 & 1,97 & 0,021 & 1,11 & - & - & - & - \\
\hline$\geq 65^{(\&)}$ & 1,00 & 1,00 & - & - & - & 1,00 & - & - & - & - \\
\hline \multicolumn{11}{|c|}{ Classe econômica } \\
\hline $\mathrm{A} / \mathrm{B}$ & 3,10 & 2,98 & 1,85 & 4,80 & 0,000 & 3,49 & 3,24 & 2,05 & 5,12 & 0,000 \\
\hline $\mathrm{C}$ & 1,26 & 1,19 & 0,86 & 1,65 & 0,298 & 1,43 & 1,36 & 1,00 & 1,85 & 0,050 \\
\hline $\mathrm{D} / \mathrm{E}^{(\&)}$ & 1,00 & 1,00 & - & - & - & 1,00 & 1,00 & - & - & - \\
\hline \multicolumn{11}{|l|}{ Sedentarismo } \\
\hline Não & 4,02 & 4,15 & 3,04 & 5,65 & 0,000 & 1,72 & 1,73 & 1,30 & 2,30 & 0,000 \\
\hline $\operatorname{Sim}^{(\&)}$ & 1,00 & 1,00 & - & - & & 1,00 & 1,00 & - & - & - \\
\hline \multicolumn{11}{|c|}{ Morbidades crônicas (Exceto Diabetes mellitus) } \\
\hline Não & 3,37 & 2,66 & 1,57 & 4,50 & 0,000 & 3,44 & 3,52 & 2,06 & 5,99 & 0,000 \\
\hline $\operatorname{Sim}^{(\&)}$ & 1,00 & 1,00 & - & - & & 1,00 & 1,00 & - & - & - \\
\hline
\end{tabular}

incisiva que aquele observado para a classe econômica (Tabela 5). Assim, o efeito do sedentarismo na QV física é cerca de duas vezes maior entre os diabéticos com OR ajustado de 4,15 (Tabela 5), quando comparado à população geral, onde o OR ajustado foi de 1,89 (Tabela 3). Além disso, verifica-se que o sedentarismo afeta de forma mais acentuada a QV física dos diabéticos (Tabela 4), enquanto em termos da população geral, este afeta mais a QV mental (Tabela 2).

$\mathrm{Na}$ população geral, "morbidades crônicas exceto DM" foi a variável que apresentou o OR de maior magnitude, tanto para o PCS (OR ajustado $=4,32)$ quanto para o MCS (OR ajustado = 2,34) (Tabela 3).

Em resumo, quanto ao perfil dos indivíduos da população geral com maior chance de ter QV acima da média, na Tabela 2, observou-se que homens, com 20 a 39 anos, casados ou em união estável, com 12 anos de estudo ou mais, pertencentes à classe $\mathrm{A} / \mathrm{B}$, não sedentários, sem morbidades crônicas e os não diabéticos também foram maioria quando considerada a pontuação do PCS e do MCS acima da média.
No modelo final da abordagem multivariada para a população em geral, a QV física (PCS) e mental (MCS) acima da média foi, ainda, explicada pelas variáveis: sexo, idade, estado civil, anos de estudo, classe econômica, sedentarismo, morbidades crônicas exceto DM e diabetes mellitus. Os homens, com maior escolaridade, pertencentes à classe $\mathrm{A} / \mathrm{B}$, não sedentários, sem morbidades crônicas e os não diabéticos tiveram maiores chances de terem alcançado uma QV física e mental acima da média (Tabela 3).

Ainda no tocante a análise da população em geral, o ajuste do modelo de regressão para o PCS mostrou-se bom, com proporção de acertos total de $72,6 \%$. Entre os positivos, o acerto foi de $78,6 \%$ para o desfecho e, entre os negativos, de $64,4 \%$. O modelo utilizado para aferir o efeito das variáveis associadas sobre o MCS apresentou proporção de acertos total de 66,3\%; entre os positivos para a ocorrência do desfecho, essa proporção foi $86,2 \%$; e, entre os negativos, foi de $32,5 \%$.

Já em relação à amostra de diabéticos, quanto à análise bivariada das variáveis de exposição e 
os desfechos PCS e MCS acima da média, todas as variáveis apresentaram associação significativa com o desfecho PCS acima da média (PCS > 42) (p-valor $<5 \%$ ), exceto raça e estado civil; já para o desfecho MCS acima da média (MCS > 47), apenas raça e idade não foram significativas (Tabela 4).

O perfil dos diabéticos que obtiveram melhor QV física na análise bivariada (Tabela 4) foi: homem; idade entre 20 e 39 anos; ter 12 anos ou mais de estudo; pertencer a classe $A / B$; não ser sedentário e não possuir outra morbidade crônica além de DM. Além destas características, estar casado ou em união promove uma melhor QV mental.

Na regressão logística multivariada com o desfecho PCS acima da média, compuseram o modelo final todas as variáveis investigadas, exceto raça e estado civil, por não terem sido significativas na análise bivariada. Após ajuste multivariado, a melhor QV física entre os diabéticos continuou associada ao sexo ("homens"), faixa etária ("20 a 39 anos"), classe econômica ("A/B"), sedentarismo ("Não") e presença de morbidade crônica exceto Diabetes mellitus ("Não"); enquanto que, no desfecho MCS acima da média, o perfil quanto à melhor QV foi o mesmo, exceto a faixa etária que apresentou p-valor > 5\% (Tabela 5).

Quanto à qualidade do ajuste por regressão logística multivariada para o desfecho PCS $>42$, observou-se a proporção de classificação total (overall) de 69,6\% - variando de 64,2\% entre os casos negativos para o desfecho e $74,7 \%$ entre os positivos. Já para o MCS $>47$, tem-se o overall de $62,5 \%$, variando de $52,7 \%$ e $71,3 \%$ entre os negativos e os positivos, respectivamente.

\section{Discussão}

Na caracterização da amostra de diabéticos, observou-se que os grupos mais vulneráveis à pior $\mathrm{QV}$, ou seja, que referiram piores escores de QV, foram mulheres, aqueles que não têm conjugue, acima de 65 anos, com baixa escolaridade e/ou rendimento, sedentários e os diabéticos com pelo menos uma outra morbidade crônica.

Neste estudo a prevalência de DM encontrada na população adulta brasileira em 2008 foi $7,5 \%$. Estudo de Viacava e Belido ${ }^{17}$ alertou para o crescimento dessa taxa no país em um período de quinze anos (1998-2013). De acordo com a IDF, a prevalência mundial de adultos com DM é de $8,3 \%$, representando aproximadamente $382 \mathrm{mi}-$ lhões de pessoas ${ }^{2}$. Na população brasileira, parte significativa com DM desconhece o diagnóstico, levando a uma possível subestimação da medida autorreferida, sendo este, na maioria das vezes, definido já na presença de complicações ${ }^{18}$.

A QV agrega a percepção subjetiva do indivíduo do bem-estar físico, emocional e social, inclui um componente cognitivo e emocional e está se tornando cada vez mais importante para os cuidados de saúde para pacientes diabéticos ${ }^{7}$. No presente estudo verificou-se que domínios referentes ao Componente físico (PCS médio $=$ 42,03) afetaram mais a QV dos pacientes com DM do que os relacionados à saúde mental (MCS médio $=47,13)$. Entende-se que o DM afete mais negativamente o bem-estar físico em decorrência das complicações agudas e crônicas e das demandas do tratamento ${ }^{7}$. Este resultado é corroborado por estudos internacionais ${ }^{19,20}$ e foi melhor que aqueles verificados nos pacientes do Centro de Diabetes Joslin em Nova Iorque ${ }^{21}$.

Estudos têm mostrado que os escores de QV para diabéticos são muito menores que os relatados para não-diabéticos ${ }^{5,22}$. A razão para escores mais baixos entre diabéticos é provavelmente multifatorial. Em comparação com os não-diabéticos, estes tendem a ser mais velhos; mais propensos ao sedentarismo; mais susceptíveis às comorbidades (como, hipertensão, doença arterial coronária, hipercolesterolemia); e a sofrer complicações crônicas como: polineuropatia, retinopatia, nefropatia, amputações, hiperglicemia e hipoglicemia. Todos estes fatores têm sido associados a uma pior $\mathrm{QV}^{6}$.

Em específico em relação ao sexo e QV, os resultados mostraram que as mulheres diabéticas possuem pior QV física e mental. Esses achados estão de acordo com a literatura ${ }^{23,24}$, existindo diferentes explicações para o mesmo: maior risco de desenvolver doença cardiovascular e/ou ser obesa ${ }^{25}$, possuir mais sintomas relacionados à hiperglicemia ${ }^{26}$, apresentar maiores taxas de depressão e ansiedade ${ }^{27}$ e maior dificuldade em fazer atividade física ${ }^{28}$. Porém, alguns estudos ${ }^{29,30}$ não verificaram associação significativa entre sexo e QV.

Quanto à idade, para a amostra total deste estudo, a média foi de $48,5 \pm 16$ anos e entre os diabéticos, observou-se média de 60,5 $\pm 13,4$ anos; com prevalência de DM de $16,7 \%$ entre aqueles com 65 anos e mais. Estes resultados são confirmados por estudos que demonstram maior prevalência de DM em pessoas com idade avançada $^{18}$, bem como dados do Ministério da Saúde ${ }^{31}$ também mostram maior número de pessoas com idade de 65 anos ou mais acometidas pelo DM. 
A piora na QV física e mental de idosos diabéticos pode ser justificada devido ao DM ser uma doença crônica que exige tratamento contínuo, e frequentemente seus portadores apresentam além das complicações próprias da doença, outras doenças que por sua vez também exigem tratamento e cuidados contínuos ${ }^{32}$. O presente estudo encontrou uma piora na chance de obter-se melhor QV, cerca de 3 vezes menor entre os de 20-39 anos quando comparados àqueles de 65 anos e mais, que está associada à perda da capacidade de execução de atividades ligadas ao trabalho ou ao cotidiano ${ }^{33}$. Para os adultos com idade inferior a 65 anos, o DM também representa sério problema, já que esses, quando se encontram em mau controle metabólico, tem que conviver durante muitos anos com as possíveis complicações decorrentes do DM, interferindo na sua expectativa e $\mathrm{QV}^{34}$.

A melhor adaptação ao diabetes mellitus tem sido associada à um maior suporte familiar ${ }^{35}$. Estudo realizado por Tavares e colaboradores evidencia que o estado civil interfere na adesão ao tratamento, na dinâmica familiar e o perfil psicossocial influencia nas variações glicêmicas, sendo o ambiente familiar um estímulo ao autocuidado ${ }^{36}$. Por outro lado, a cor da pele não tem sido associada ao relato de DM, sendo entretanto classificado com um fator de risco não modificável (herança genética) ${ }^{37}$.

Percebe-se pior QV entre os diabéticos analfabetos, com um gradiente crescente de chance de melhor QV conforme o aumento da escolaridade. Este fato pode estar relacionado diretamente ao déficit de conhecimento sobre a doença entre os diabéticos de baixa escolaridade, um dos principais problemas para a não realização/ adesão do tratamento de forma adequada. A democratização do conhecimento sobre o processo saúde-doença, principalmente no âmbito das doenças crônicas, é fundamental para obtenção de resultados satisfatórios no tratamento, além de possibilitar a escolha por hábitos de vida saudáveis, contribuindo assim para o aumento da $\mathrm{QV}^{38}$. Estes resultados estão de acordo com os obtidos por Eren e colaboradores (2008), que apontaram em seu estudo a associação entre escolaridade e melhor QV em pacientes com DM tipo $2^{39}$.

No Brasil observa-se que o número de pessoas que referem problemas de saúde diminui à medida que a renda aumenta, o que coloca $o$ país em um padrão de grandes diferenças sociais em termos de saúde ${ }^{3}$. No presente estudo, verificou-se este mesmo fenômeno, onde a chance de obter-se uma melhor QV, principalmente entre os diabéticos, aumenta cerca de três vezes mais quando se considera os mais altos níveis de classe econômica. Desta forma, infere-se que uma maior renda atuaria como fator facilitador na adesão/adequação ao tratamento do diabetes mellitus $^{40}$, bem como em sua prevenção, uma vez que o DM é uma doença onerosa para o indivíduo e para o sistema de saúde em geral. Segundo a IDF, em 2015 o gasto anual com DM foi 673 bilhões de dólares, representando $12 \%$ do gasto global em saúde 2 . Nos Estados Unidos o custo de um indivíduo com a doença foi estimado em três vezes mais quando comparado a um sem a doença. No Brasil, o valor estimado para os custos diretos com a doença chega a 3,9 bilhões de dólares por ano ${ }^{41}$.

Outro importante aspecto oriundo do estudo é o relevante papel desempenhado pelo sedentarismo na $\mathrm{QV}$, seja no surgimento ou no desenvolvimento do DM. Verificou-se quatro vezes mais chance de obter-se uma pior QV entre os diabéticos sedentários quando comparado aos não sedentários, principalmente na QV física. Ressaltase ainda neste estudo o aumento da prevalência de diabéticos entre os sedentários (11\%), quando comparado a prevalência de DM na população geral $(7,5 \%)$. Além disso, sabe-se que no tratamento do DM é fundamental não apenas o uso de medicamentos, mas também a mudança no estilo de vida ${ }^{38}$, uma vez que as doenças crônicas, como as cardiopatias, diabetes mellitus e hipertensão, apresentam elevado índice de mortalidade e estão associadas aos hábitos e dietas inadequadas ${ }^{42}$.

A literatura demonstra que aqueles que são diabéticos e possuem outras doenças crônicas têm piores escores de QV do que os sujeitos portadores exclusivamente do $\mathrm{DM}^{6,43}$. Além disso, no presente estudo, observa-se que diabéticos com outras comorbidades apresentaram cerca de três vezes menos chance de ter melhor QV. Sendo assim, o número de morbidades crônicas exerce efeitos negativos na QV da população em geral e em específico entre os diabéticos ${ }^{44}$.

Os resultados expostos e discutidos elucidam, então, a associação entre diabetes mellitus e qualidade de vida. Porém, é importante ressaltar as limitações relacionadas ao desenho epidemiológico empregado, uma vez que os estudos transversais são restritos à identificação de associações, impossibilitando o estabelecimento da causalidade na relação encontrada, além de não permitir a realização de análises de temporalidade entre exposição e desfecho, não podendo assim apontar fatores de risco. 
Outra limitação diz respeito ao diagnóstico de DM autorreferido. Apesar de apresentar concordância com a verdadeira ocorrência da doença, a grande proporção de indivíduos que desconhece ser portadora desse agravo pode levar a uma importante subestimação dos casos de diabetes mellitus encontrados na população aqui estudada. Estimativas apontam que no mundo, de 25 a $50 \%$ dos indivíduos desconhecem que tem a doença ${ }^{2}$. A acurácia da informação referida de morbidade varia conforme a doença, sua gravidade, características sociodemográficas e econômicas. Entretanto, apesar das limitações, diversos autores concordam que o uso dessa metodologia vem crescendo mundialmente devido à sua praticidade e baixo custo ${ }^{43,45}$.

Por outro lado, como forças do estudo, a base de dados populacional da PDSD traz informações agregadas de morbidades crônicas e fatores associados em relação a QV, mensurada por instrumento com confiabilidade comprovada em amostras heterogêneas de indivíduos e pacientes diabéticos ${ }^{46}$.

Este artigo enfatiza especificamente a presença do DM e seus prejuízos na QV, o que demonstrou ser uma lacuna na literatura, principalmente, nacional. Assim, é possível evocar-se questões que acometem os diabéticos, além de encontrar caminhos para promoção de saúde, redução de danos e outros fatores que melhoram sua QV.

\section{Conclusão}

O presente trabalho traçou o perfil dos indivíduos no que tange aos fatores relacionados à chan- ce de uma melhor QV, seja em uma amostra da população brasileira ou em portadores de DM, fornecendo subsídios para a atenção à saúde. Tal avaliação contribui para compreenderem-se determinados fatores presentes no cotidiano dos indivíduos em geral, e em particular dos diabéticos, que interferem no acompanhamento e na adesão ao tratamento do DM e, por vezes, estão encobertos nas relações familiares, profissionais e sociais.

Os resultados desse estudo mostraram o impacto negativo do DM na QV dos participantes, perceptível pelos escores médios obtidos nos domínios físico e mental do SF-36, principalmente na QV física. O perfil de diabéticos que apresentaram maiores chances de uma pior QV física e mental foram as mulheres, sedentárias, com 65 anos ou mais, pertencentes a classe D/E e com outras morbidades crônicas. Para a população geral além desses fatores, não ter um companheiro e ser analfabeto proporcionou uma pior QV.

Dentre as variáveis analisadas, verificou-se que ser sedentário e pertencer à classe $\mathrm{D} / \mathrm{E}$ exerceram maior influência na chance de obter-se pior QV, principalmente entre diabéticos quando comparado à população geral, com cerca de duas vezes mais chances. Assim, tais aspectos evidenciam-se como importantes fatores a serem considerados na gênese e evolução dessa morbidade.

Dessa forma, conhecer os fatores que afetam a QV da população brasileira, bem como os efeitos do diabetes mellitus, possibilitam o planejamento de ações de promoção da saúde e prevenção voltadas a este grupo, de maneira a capacitá-lo para escolhas saudáveis em seu cotidiano, com vista à melhoria da QV. 


\section{Colaboradores}

RLB Santos e MR Campos participaram da concepção e delineamento, redação, análise dos dados e revisão do artigo e LS Flor participou da redação e revisão do artigo.

\section{Referências}

1. Sociedade Brasileira de Diabetes (SBD). Diretrizes da Sociedade Brasileira de Diabetes (2015-2016). São Paulo: SBD; 2016.

2. International Diabetes Federation (IDF). Diabetes Atlas. 7a ed. Brussel: IDF; 2015.

3. Instituto Brasileiro de Geografia e Estatística (IBGE). Pesquisa Nacional de Saúde 2013. Acesso e utilização dos serviços de saúde, acidentes e violências Brasil, Grandes Regiões e Unidades da Federação. Rio de Janeiro: IBGE; 2015.

4. Brasil. Ministério da Saúde (MS). Vigitel Brasil 2013: vigilância de fatores de risco e proteção para doenças crônicas por inquérito telefônico. Brasília: MS; 2014.

5. Imayama I, Plotnikoff RC, Courneya KS, Johnson JA. Determinants of quality of life in adults with type 1 and type 2 diabetes. Health Qual Life Outcomes 2011; $9(1): 115$.

6. Venkataraman K, Wee HL, Leow MKS. Associations between complications and health-related quality of life in individuals with diabetes. Clin Endocrinol (Oxf) 2013; 78(6):865-873.

7. Kazemi-Galougahi M, Ghaziani HN, Ardebili HE, Mahmoudi M. Quality of life in type 2 diabetic patients and related effective factors. Indian J Med Sci 2012; 66(9):230.

8. Laguardia J, Campos MR, Travassos CM, Najar AL, Anjos LA, Vasconcellos MM. Psychometric evaluation of the SF-36 (v.2) questionnaire in a probability sample of Brazilian households: results of the survey Pesquisa Dimensões Sociais das Desigualdades (PDSD), Brazil, 2008. Health Qual Life Outcomes 2011; 9(1):61.

9. Centro de Estudos da Metrópole. Banco de dados da Pesquisa Dimensões Sociais da Desigualdade (PDSD). São Paulo: USP; 2016. [acessado 2016 nov 12]. Disponível em: http://www.fflch.usp.br/centrodametropole/ $\mathrm{v} 3 /$ bases.php? retorno $=$ 716\&language $=$ pt_br

10. Ciconelli R, Ferraz M, Santos W, Meinão I, Quaresma M. Tradução para a língua portuguesa e validação do questionário genérico de avaliação da qualidade de vida SF-36 (Brasil SF-36). Rev Bras de Reumatologia 1999; 39(3):143-150.

11. Gorenstein G, Wang Y, Hungerbühler I. Instrumentos de avaliação em saúde mental. Porto Alegre: Artmed Ltda.; 2016.

12. Lopes AD, Ciconelli RM, Reis FB. Medidas de avaliação de qualidade de vida e estados de saúde em ortopedia. Rev Bras Ortop 2007; 42(12):355-359.

13. Ware JE, Sherbourne CD. The MOS 36-item shortform health survey (SF-36). I. Conceptual framework and item selection. Med Care 1992; 30(6):473-483.

14. Associação Brasileira de Empresas de Pesquisa (ABEP). Critério padrão de classificação econômica Brasil/2008. São Paulo: ABEP; 2007.

15. Hallal PC, Victora CG, Wells JCK, Lima RC. Physical inactivity: prevalence and associated variables in Brazilian adults. Med Sci Sports Exerc 2003; 35(11):18941900.

16. SPSS Inc. SPSS Statistics for Windows, Version 17.0. Chicago: SPSS Inc; 2008.

17. Viacava F, Bellido JG. Condições de saúde, acesso a serviços e fontes de pagamento, segundo inquéritos domiciliares. Cien Saude Colet 2016; 21(2):351-370. 
18. Leal LB, Moura IH, Carvalho RBN, Leal NTB, Silva AQ, Silva ARV. Quality of life regarding the health of people with diabetes mellitus type 2. Rev Rede Enferm Nordeste 2014; 15(4):676-682.

19. Goldney RD, Phillips PJ, Fisher LJ, Wilson DH. Diabetes, depression, and quality of life: a population study. Diabetes Care 2004; 27(5):1066-1070.

20. Gulliford MC, Mahabir D. Relationship of health-related quality of life to symptom severity in diabetes mellitus: a study in Trinidad and Tobago. J Clin Epidemiol 1999; 52(8):773-780.

21. Trief PM, Wade MJ, Pine D, Weinstock RS. A comparison of health-related quality of life of elderly and younger insulin-treated adults with diabetes. Age Ageing 2003; 32(6):613-618.

22. Urzúa MA, Chirino A, Valladares G. Autoreporte de la calidad de vida relacionada con la salud en diabetes mellitus tipo 2. Rev Médica Chile 2011; 139(3):313-320.

23. Bosić-Zivanović D, Medić-Stojanoska M, Kovacev-Zavisić $B$. The quality of life in patients with diabetes mellitus type 2. Vojnosanit Pregl 2012; 69(10):858-863.

24. Naughton MJ, Yi-Frazier JP, Morgan TM. Longitudinal Associations between Sex, Diabetes Self-Care, and Health-Related Quality of Life among Youth with Type 1 or Type 2 Diabetes Mellitus. J Pediatr 2014; 164(6):1376-1383.

25. Norhammar A, Schenck-Gustafsson K. Type 2 diabetes and cardiovascular disease in women. Diabetologia 2013; 56(1):1-9.

26. Legato MJ, Gelzer A, Goland R. Gender-specific care of the patient with diabetes: review and recommendations. Gend Med 2006; 3(2):131-158.

27. Eiser JR, Riazi A, Eiser C, Hammersley S, Tooke JE. Predictors of psychological well-being in types 1 and 2 diabetes. Psychol Health 2001; 16(1):99-110.

28. Wändell PE. Quality of life of patients with diabetes mellitus. An overview of research in primary health care in the Nordic countries. Scand J Prim Health Care 2005; 23(2):68-74.

29. Johnson JA, Nowatzki TE, Coons SJ. Health-related quality of life of diabetic Pima Indians. Med Care 1996; 34(2):97-102.

30. Ucan O, Ovayolu N. Relationship between diabetes mellitus, hypertension and obesity, and health-related quality of life in Gaziantep, a central south-eastern city in Turkey. J Clin Nurs 2010; 19(18):2511-2519.

31. Brasil. Ministério da Saúde (MS). Estratégias para o cuidado da pessoa com doença crônica: diabetes mellitus. Brasília: MS; 2013. (Cadernos de Atenção Básica, n. 36).

32. Speight J, Sinclair AJ, Browne JL, Woodcock A, Bradley C. Assessing the impact of diabetes on the quality of life of older adults living in a care home: validation of the ADDQoL Senior: Quality of life in older adults: validation of the ADDQoL Senior. Diabet Med 2013; 30(1):74-80.

33. Otero LM, Zanetti ML, Teixeira CRS. Sociodemographic and clinical characteristics of a diabetic population at a primary level health care center. Rev Lat Am Enfermagem 2007; 15(1):768-773.

34. Ferreira FS, Santos CB. Qualidade de vida relacionada à saúde de pacientes diabéticos atendidos pela Equipe de Saúde da Família. Rev enferm UERJ 2009; 17(3):406411.
35. August KJ, Rook KS, Franks MM, Parris Stephens MA. Spouses' involvement in their partners' diabetes management: Associations with spouse stress and perceived marital quality. J Fam Psychol 2013; 27(5):712-721.

36. Tavares DMS, Rodrigues FR, Silva CGC, Miranzi SSC. Caracterização de idosos diabéticos atendidos na atenção secundária. Cien Saude Colet 2007; 12(5):13411352.

37. Barreto SM, Figueiredo RC de. Doença crônica, auto-avaliação de saúde e comportamento de risco: diferença de gênero. Rev Saude Publica 2009; 43(1):38-47.

38. Jain V, Shivkumar S, Gupta O. Health-related quality of life (Hr-Qol) in patients with type 2 diabetes mellitus. North Am J Med Sci 2014; 6(2):96-101.

39. Eren I, Erdi O, Sahin M. The effect of depression on quality of life of patients with type II diabetes mellitus. Depress Anxiety 2008; 25(2):98-106.

40. Guimarães FPM, Takayanagui AMM. Orientações recebidas do serviço de saúde por pacientes para o tratamento do portador de diabetes mellitus tipo 2. Rev Nutr 2002; 15(1):37-44

41. Sociedade Brasileira de Diabetes (SBD). Cuidados de Enfermagem em Diabetes Mellitus. São Paulo: SBD; 2009.

42. Barbosa Filho VC, Campos W, Lopes AS. Epidemiology of physical inactivity, sedentary behaviors, and unhealthy eating habits among brazilian adolescents: a systematic review. Cien Saude Colet 2014; 19(1):173194.

43. Lima-Costa MF, Peixoto SV, Firmo JOA, Uchoa E. Validade do diabetes auto-referido e seus determinantes: evidências do projeto Bambuí. Rev Saude Publica 2007; 41(6):947-953.

44. Bourdel-Marchasson I, Druet C, Helmer C. Correlates of health-related quality of life in French people with type 2 diabetes. Diabetes Res Clin Pract 2013; 101(2):226-235.

45. Iser BPM, Malta DC, Duncan BB, de Moura L, Vigo A, Schmidt MI. Prevalence, correlates, and description of self-reported diabetes in brazilian capitals - results from a telephone survey. PloS One 2014; 9(9):e108044.

46. Norris SL, McNally TK, Zhang X. Published norms underestimate the health-related quality of life among persons with type 2 diabetes. J Clin Epidemiol 2011; 64(4):358-365.

Artigo apresentado em 11/11/2016

Aprovado em 04/03/2017

Versão final apresentada em 06/03/2017 